\title{
Breast Volume Measurements Using 3-D Surface Imaging: A Standardized Validation of the Introduced Method and a Comparison to Classical Approaches
}

\author{
Stefan RAITH, Maximilian EDER, Fee ARMBRECHT, Alexander VOLF, and Laszlo KOVACS* \\ Research Group Computer Aided Plastic Surgery - CAPS, \\ Clinic for Plastic Surgery and Hand Surgery, Technical University Munich, Germany
}

\begin{abstract}
Introduction: Precise and objective calculation of breast volume can be helpful to evaluate the aesthetic result of breast surgery, but traditional methods are unsatisfactory. Three-dimensional (3-D) scanning of the body surface allows reproducible and objective assessment of the complex breast region but requires further investigation before clinical application. The main goal of this study was to investigate the precision and accuracy of breast volume measurement using 3-D body scanning and to compare breast volume calculation with 3-D scanning and three classic methods, focusing on relative advantages, disadvantages, and reproducibility.
\end{abstract}

Materials and Methods: Five independent observers standardized the 3-D scanning method using two dummy models $(n=200)$ and examined its applicability with 6 test subjects and 10 clinical patients $(n=2220)$. Breast volume measurements obtained with the 3-D scanner technology were compared with reference measurements obtained from test subjects through MRI, thermoplastic castings and anthropomorphic measurements.

Results: The mean deviation of the breast volume measurements of one test subject by all observers, expressed as percentage of volume, was $2.86+/-0.98$, significantly higher than the deviation for the dummy models, $1.65+/-0.42(p<0.001)$. Inter-observer differences in measurement precision were not statistically significant. The mean breast volumes obtained by MRI (441.42 +/- $137.05 \mathrm{cc})$ and 3-D scanning $(452.51+/-141.88 \mathrm{cc})$ significantly correlated $(r=0.995, p<0.001)$. MRI showed the highest measurement precision, with a mean deviation (expressed as percent of mean breast volume) of 1.56 +/- 0.52 compared to $2.27+/-0.99$ for the 3D scanner, $7.97+/-3.53$ for thermoplastic castings, and $6.26+/-1.56$ for the anthropomorphic measurements. Breast volume calculations using MRI showed the best agreement to 3-D scanning measurement $(r=0.990)$, followed by anthropomorphic measurement $(r=0.947)$, and thermoplastic castings $(r=0.727)$.

Conclusion: Breast volume measurement using 3-D surface imaging represents a sufficiently precise and accurate method to guarantee objective and exact recording. Compared to three classical methods for breast volume calculation, 3D scanning allows precise measurement, better spatial interpretation of the anatomical area to be operated on (due to lack of chest deformation), non-invasiveness, and good patient tolerance.

Keywords: breast volume measurements, standardization, 3-D scan

\footnotetext{
*I.kovacs@Irz.tum.de
} 

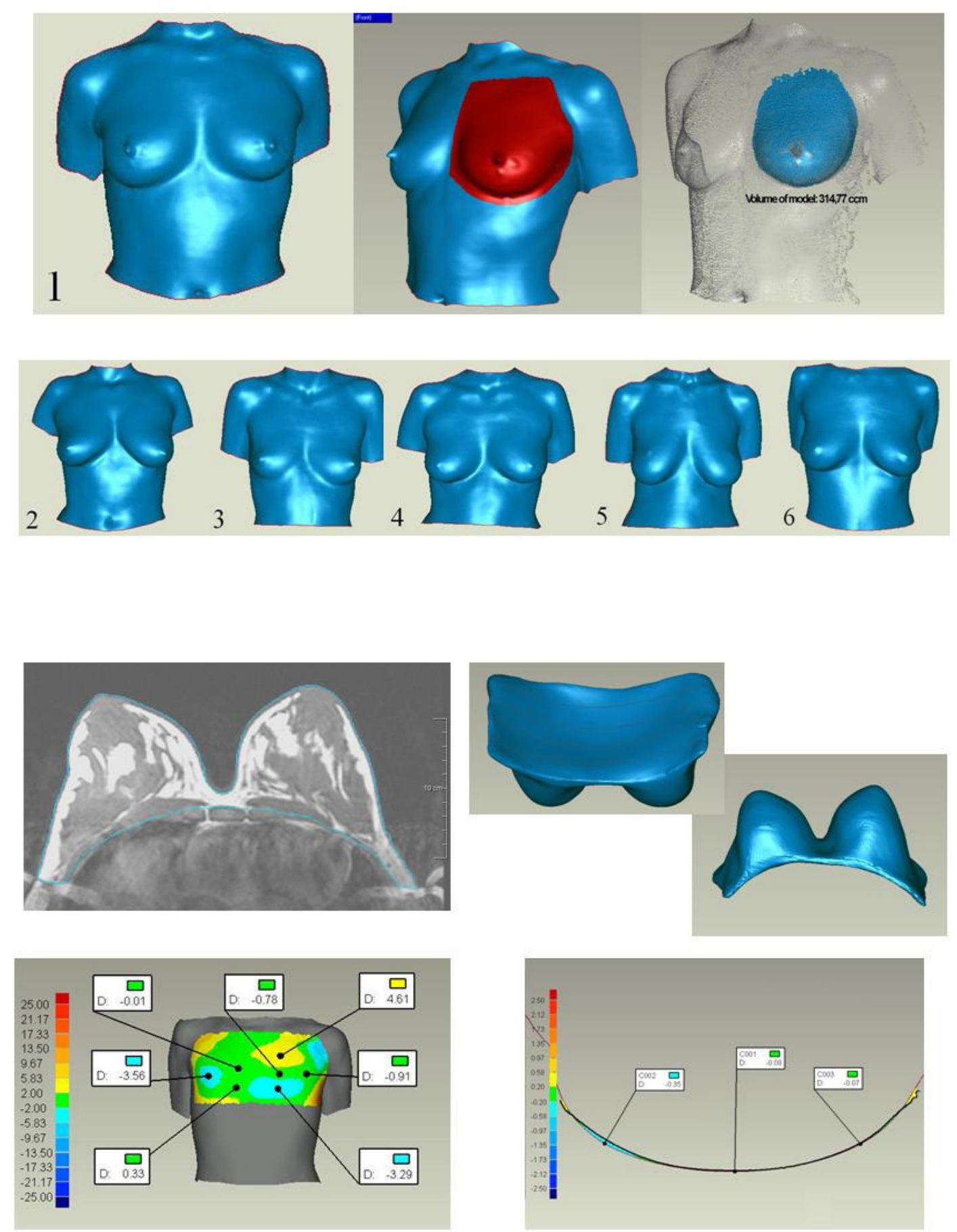